\title{
On Exploiting Spatial Correlation For Energy Harvesting Wireless Sensor Networks
}

\author{
Ali Al-QAMAJI \\ Department of Electrical - Electronics Engineering \\ İzmir Institute of Technology \\ İzmir, Turkey \\ alialqamaji@iyte.edu.tr
}

\author{
Barış ATAKAN \\ Department of Electrical - Electronics Engineering \\ İzmir Institute of Technology \\ İzmir, Turkey \\ barisatakan@iyte.edu.tr
}

\begin{abstract}
Wireless Sensor Network (WSN) is a set of inexpensive densely deployed sensor nodes with limited functionalities and scarcity in energies. The observations of sensors are forwarded directly to the Base Station (BS). In densely deployed sensors, sensing data are likely to be highly correlated in space domain, which produces unfavorable redundant readings and wasting in energy. In this paper, we propose an Event DistortionBased Node Selection (EDNS) algorithm which exploits spatial correlation for reducing inessential sensor nodes that have correlated readings for improving Energy-Efficiency (EE) with acceptable distortion level. Furthermore, we derive a theoretical framework of distortion function for single-hop communication model to observe the advantages from energy harvesting to the accuracy level. Furthermore, the trade-off between energy consumption and distortion level is investigated.
\end{abstract}

Index Terms-Wireless Sensor Networks (WSNs), Spatial correlation, Distortion, Energy-Efficient, Energy Harvesting.

\section{INTRODUCTION}

$\mathrm{I}^{\mathrm{N}}$ recent years, the engineers have tried to develop and optimize Wireless Sensor Networks (WSNs) to be used in civil and military applications [1]. WSNs can be defined as a set of sensor nodes responsible for sensing a physical phenomena, and forwarding their observations to the sink node. Furthermore, saving energy is one of the main concerns of protocols and applications in WSNs. Also, adding harvesting units to the sensor nodes are successfully addressed the challenges of charging the battery-powered sensors. For harvesting sensors [2], there are many available sources to be harvested, e.g., sun, winds, Rf-Signals, etc. In this paper, we used Rf-energy harvesting model.

In WSNs, nodes deployment might be random or systematic [1]. Random deployment has an advantage of being less expensive than fixed deployment, but it has a coverage problem [1]. Hence, this issue can be addressed by increasing the node density in an event area. However, in densely deployment case, nearby sensors get similar observations (spatially correlated) [3]. By keeping the distortion below a predefined threshold, sensors can exploit spatial correlation to reduce the forwarded data to the sink node. This reduction improves the EnergyEfficiency (EE) as well as the distortion level is maintained.

In this paper, we propose an Event Distortion-Based Node Selection (EDNS) algorithm to reduce the energy consumption

978-1-5090-6494-6/17/\$31.00 2017 IEEE with a satisfactory level of distortion. The remaining part is organized as follows. The Section II covers related works. In Sections III and IV the network model and an on-off algorithm are presented, respectively. The simulation results of the EDNS algorithm is given in Section V with conclusion in Section VI.

\section{RELATED WORKS}

One of the main performance criteria in WSNs is distortion level and energy consumption. The distortion represents a similarity level between original and estimated readings. The distortion function is derived in [3] and [4] with minimum mean squared sense. In [3] single-hop model is used without channel noise, while [4] uses the same model but with one noise term at the sink node. Besides, in [3], the exponential model is used as spatial correlation between sensors, but in [4], the readings of sensors are assumed to be identical.

In [3], Iterative Node Selection (INS) algorithm is presented to exploit spatial correlation for selecting a representative nodes, i.e., the only nodes that requires to transmit data to the sink node, using vector quantization. However, the INS algorithm prevents the remaining nodes from observing and forwarding duties. In this paper, we propose the EDNS algorithm by exploiting spatial correlation, but here we exploit both representative and unrepresentative nodes, unlike [3]. Furthermore, in the INS algorithm, the observation noise is only considered to derive the distortion function for WSNs. However, in addition to the observation noise, the channel also needs to be considered in the distortion function. Here, we take the observation and the channel noise into account for deriving the distortion function in energy-harvesting WSNs.

\section{NETWORK MODEL}

Sensor nodes are deployed in the event area for observing the physical phenomena (i.e., point source at $n_{0}$ ) and then they forward their data directly by single-hop links to the sink node. For $N$ sensors, each sensor gets the noisy version of original source event, $S$, which is given by

$$
X_{i}=S_{i}+N_{i}
$$

where $N_{i}$ is an observation noise for sensor node $n_{i}$, which is modeled as i.i.d. Gaussian random variables with zero mean 


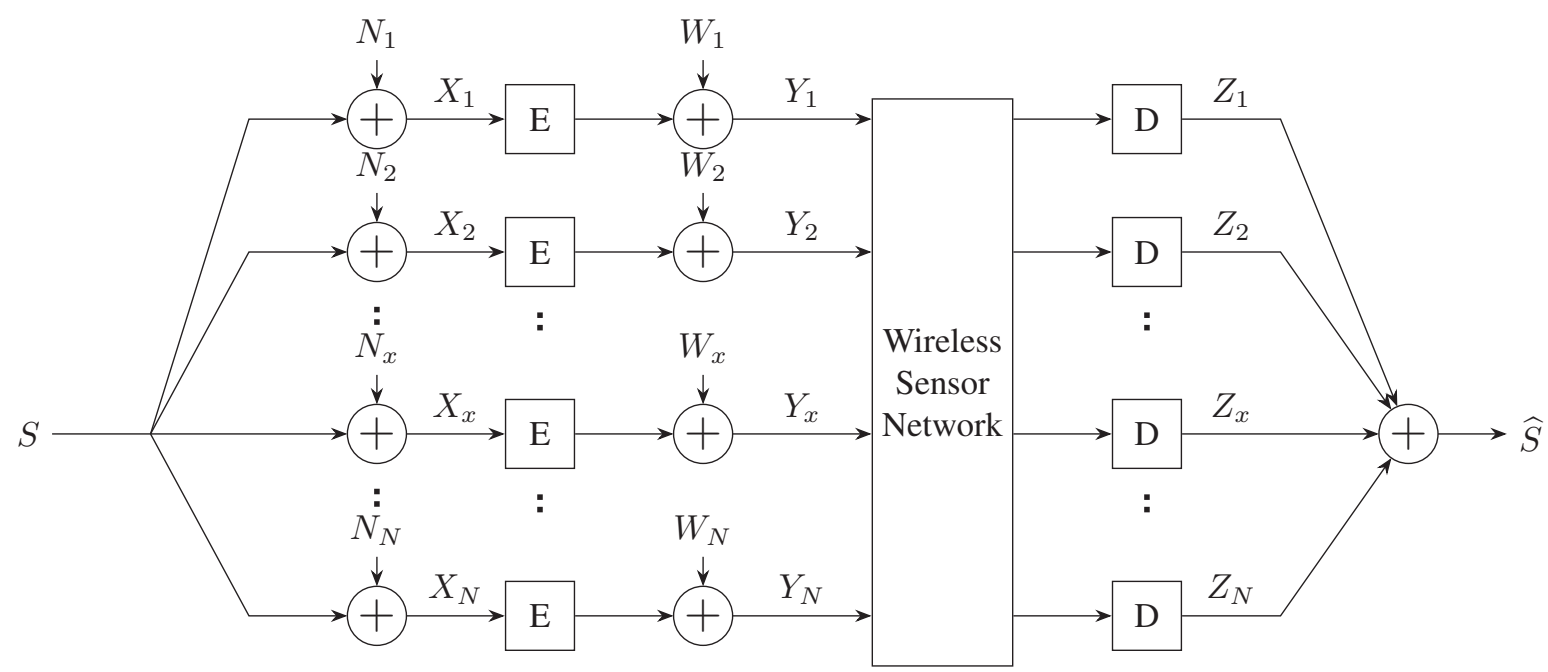

Fig. 1: Point-to-Point Network Model

and variance $\sigma_{N}^{2}$. The event, $S_{i}$, is modeled as joint Gaussian random variables (JGRVs) as

$$
E\left[S_{i}\right]=0, \quad \operatorname{Var}\left[S_{i}\right]=\sigma_{S}^{2}, \quad i=1,2, \ldots, N
$$

The correlation model is power exponential model, as [3]

$$
\rho(i, j)=\frac{E\left[S_{i} S_{j}\right]}{\sigma_{S}^{2}}=e^{\left(d(i, j) / \theta_{1}\right)^{\theta_{2}}}
$$

where $\rho(i, j)$ and $d(i, j)$ are the correlation coefficient and distance, between node $n_{i}$, and node $n_{j}$, respectively. The parameter $\left(\theta_{2}=1\right)$ is for exponential model, and $\theta_{1}$ is the controlling parameter for the correlation range between sensors.

\section{EVENT DISTORTION-BASED NODE SELECTION (EDNS)}

Here, we present the EDNS algorithm. It is an off-line algorithm which runs at the sink node to reduce the sensor nodes so as to determine which sensors transmit their information to the sink node for an acceptable level of event signal reconstruction distortion. The distortion function is derived for single-hop model. Then, the algorithm is presented.

\section{A. Distortion with Point-To-Point Communication}

All sensors transmit their readings to the sink node as in Fig 1. The sink node is then calculates the point distortion, $D_{\text {Point }}$. Since the uncoded transmission is optimal for singlehop case with Gaussian source [4], each sensor forwards a noisy version, $X_{i}$, of original data without encoding [3]. Hence, the transmitted signal, $Y_{i}$, is a scaled version of $X_{i}$, added with channel noise, $W_{i}$. Then, $Y_{i}$ is written as

$$
Y_{i}=\alpha X_{i}+W_{i} \text {, where } \alpha=\sqrt{\frac{P}{\sigma_{S}^{2}+\sigma_{N}^{2}}}
$$

The channel noise, $W_{i}$, is modeled as i.i.d. Gaussian r.vs with zero mean and variance $\sigma_{W}^{2}$. The scalar, $\alpha$, is determined with respect to the power constraints, $P$. The estimation of received signal at the sink node, $Z_{i}$, is estimated by a Minimum Mean Squared Error (MMSE) estimator. Then, by averaging all $Z_{i}$, the estimated version, $\hat{S}$, is stated as

$$
\hat{S}=\frac{1}{N} \sum_{i=1}^{N} \frac{\alpha \sigma_{S}^{2}}{P+\sigma_{W}^{2}} Y_{i}
$$

Finally, Point Distortion, $D_{\text {point }}$, is computed as

$$
\begin{aligned}
D_{\text {point }}= & E\left[(S-\hat{S})^{2}\right]=\sigma_{S}^{2}-\frac{\sigma_{S}^{4}}{N^{2}} \sum_{i=1}^{N} \frac{\alpha}{P+\sigma_{W}^{2}} \\
& \left(2 N \rho\left(S, S_{i}\right)-1-\sum_{j \neq i}^{N} \frac{\alpha^{2} \rho\left(S_{i}, S_{j}\right)}{\left(P+\sigma_{W}^{2}\right)}\right)
\end{aligned}
$$

where $\rho\left(S, S_{i}\right)$ and $\rho\left(S_{i}, S_{j}\right)$ are the correlation coefficients between $n_{0}$ and $n_{i}$, and between $n_{i}$ and $n_{j}$, respectively.

\section{B. EDNS Algorthim}

According to the preceding results, the EDNS algorithm is presented. The EDNS algorithm requires to select a smaller set of sensors, rather than all sensors. These selected sensors are determined through exploiting spatial correlation such that the acceptable level of event signal reconstruction distortion is maintained at the sink node. The EDNS algorithm uses Vector Quantization (VQ) as a way to exploit spatial correlation.

The VQ design expresses as follows [5]: Given source vector with its statistical properties, distortion constraint, and the number of codewords. Then, VQ algorithm aims to find the codebook and partitions (i.e., voronoi regions), which results in the smallest satisfactory average distortion. More specifically, the VQ algorithm tries to represent all possible codewords in code space by a subset of codewords, within the distortion constraint (i.e., maximum acceptable distortion). Hence, the spatial correlation is exploited using VQ, where all sensor nodes in an event area are need to be represented with smaller 


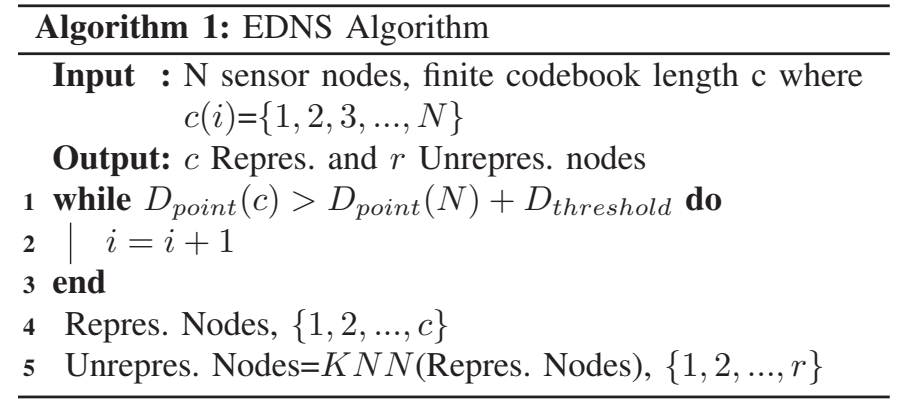

number. The VQ algorithm, when it applies to the sensor nodes selection issue, the codebook and partitions can be determined. Since the sensor nodes are densely deployed, it is possible to refer the closest nodes to the locations of codebook as representative nodes, $c$, while the remaining nodes are refer as unrepresentative nodes, $r$.

The EDNS algorithm starts with selecting one codebook as an input to VQ, as well as all $N$ sensor nodes as a source vector. The outputs are one representative node and set of unrepresentative nodes. Then, the EDNS algorithm iteratively increases the number of representative nodes (i.e., by increasing the codebook). For each increment, the representative nodes increase. The algorithm continues to increase the number of representative nodes until their point distortion (6), $D_{\text {point }}(c)$, achieves a distortion constraint. Then, the K-Nearest Neighbor (KNN) [6] is used to divide the unrepresentative nodes into a subsets with respect to their voronoi diagrams.

The EDNS algorithm only requires the readings of representative nodes to be sent to the sink node. If the representative node has no sufficient energy, the EDNS algorithm replaces the readings of representative node with the readings of the subset of unrepresentative nodes which they locate inside its voronoi cell, until its energy level is recovered by harvesting.

\section{Simulations}

In this section, the EDNS algorithm conducted by MATLAB to define its detailed performance. The simulation applies in terms of distortion, energy consumption and network lifetime, for both harvesting and non-harvesting cases. A total of 100 sensor nodes are deployed randomly in $100 \times 100 \mathrm{~m}^{2}$ event area. The sink node locates at the center of event area. Furthermore, a cell tower locates at the center of event area, i.e., GSM900, [7] with transmitting and receiving antenna gains $(17 \mathrm{~dB}, 9 \mathrm{~dB})$ and center frequency is $950 \mathrm{MHZ}$. The cell tower is used as a renewable energy source for all sensor nodes, with transmitting power 20 watts. The harvesting energy is calculated by friis equation for free path loss. In this paper, we follows the the energy model of [8] with $E_{\text {elec }}=50$ $\mathrm{nJ} / \mathrm{bit}, \epsilon_{f s}=10 \mathrm{PJ} / \mathrm{bit} / \mathrm{m}^{2}$, packet size length is 500 byte, and initial energy is $0.5 \mathrm{~J}$.

\section{A. Performance of the EDNS Algorithm}

Initially, the sink node allocates all sensors and estimates their received samples based on the single-hop communication and determines their point distortion for all $N$ sensor nodes, $D_{\text {point }}(N)$. Then, the sink node applies Vector Quantization
(VQ). The VQ initiates with all sensors as vector source and the code vector is one. According to the EDNS algorithm, the sink node starts to increase the number of code word until the representative nodes achieve the distortion constraints. The maximum achievable distortion is given by

$$
D_{\text {point }}(N)+D_{\text {threshold }} \geqslant D_{\text {constraint }}
$$

where $D_{\text {Point }}(N)$ is the minimum distortion [3]. The threshold distortion, $D_{\text {thresold }}$, equals to 0.001 for noiseless $\left(\sigma_{W}^{2}=0\right)$ and noisy $\left(\sigma_{W}^{2}=0.5\right)$ terms.

After repeating the experiment over 100 trials (Monte Carlo), the EDNS nearly selects 84 unrepresentative nodes and 16 representative nodes, as in Fig 2a. Then, the representative nodes are responsible for their voronoi diagrams and each unrepresentative node belongs to specific digram, which it is defined by applying $K N N$ [6] as in Fig $2 \mathrm{~b}$.

After both representative and unrepresentative nodes are determined, the EDNS algorithm is settled. Then, we compares between the EDNS algorithm, INS algorithm [3] and the general case, i.e., all $N$ sensors. The comparison obtains with and without harvesting. For non-harvesting case, the EDNS algorithm is initially uses the representative nodes, hence its performance is similar to the INS algorithm, where the

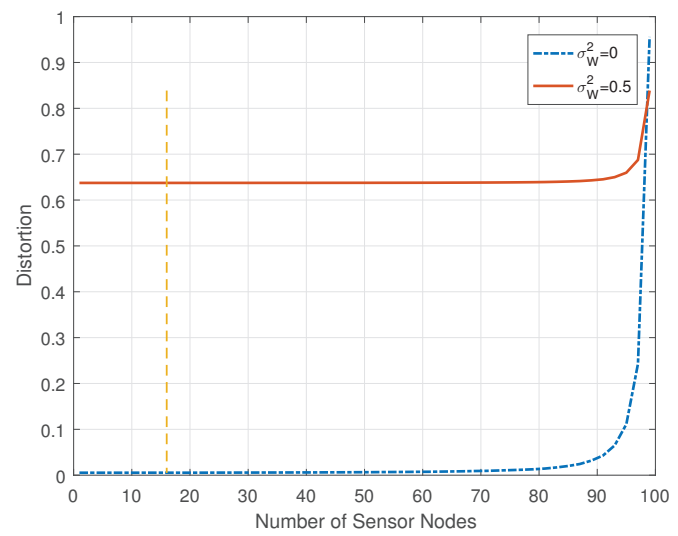

(a) Vector Quantization

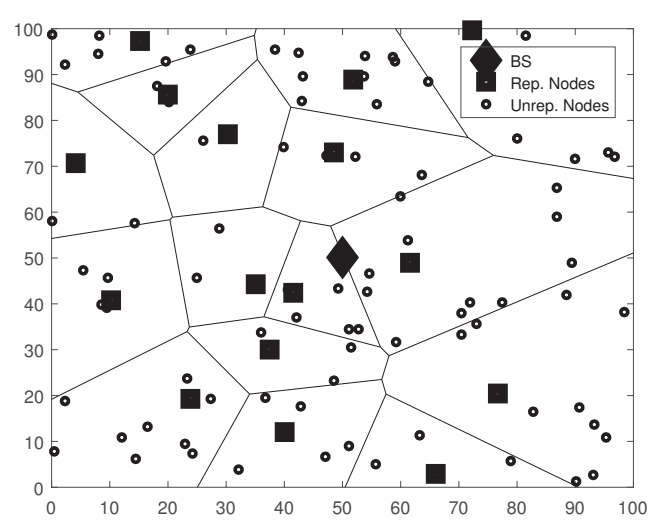

(b) Resulted Network

Fig. 2: Exploiting Spatial Correlation 


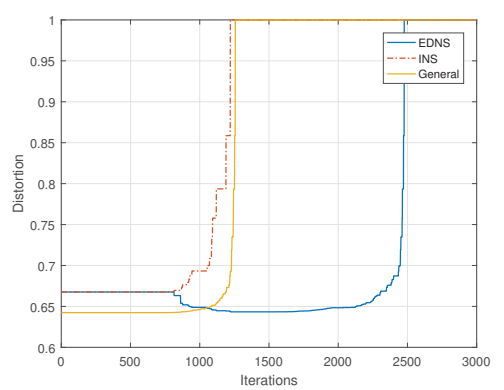

(a) Distortion for Non-harvesting.

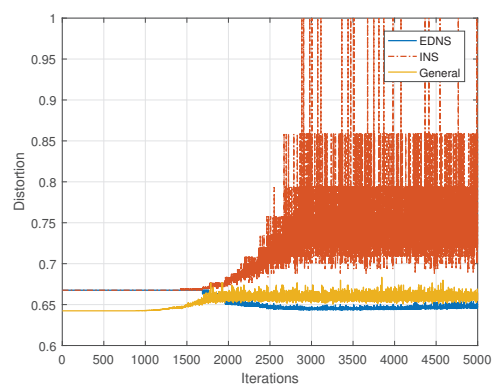

(d) Distortion for Harvesting.

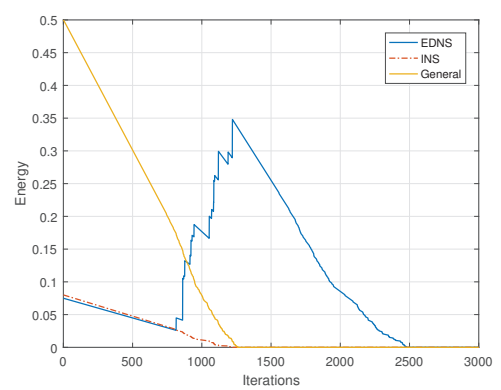

(b) Energy for Non-harvesting.

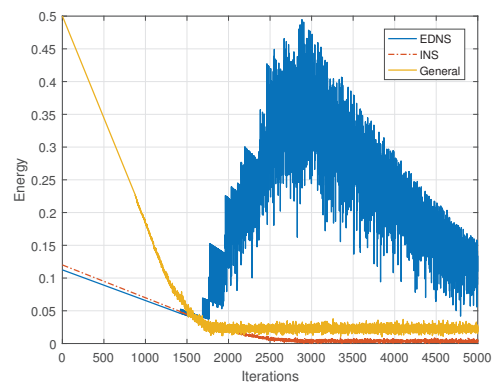

(e) Energy for Harvesting.

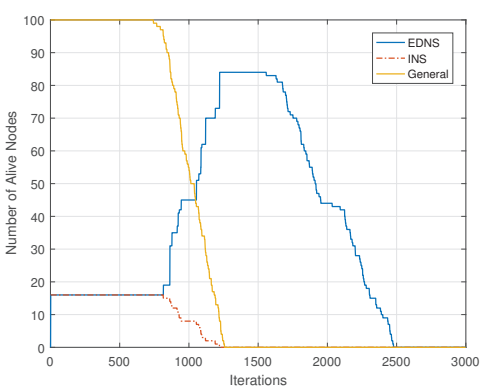

(c) Number of Alive Nodes for Non-harvesting.

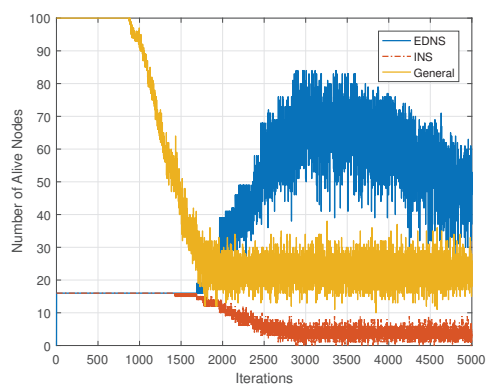

(f) Number of Alive Nodes for Harvesting.

Fig. 3: Harvesting and Non-harvesting Cases

INS algorithm also uses representative nodes. However, the distortion with the INS algorithm is lifted up as shown in Fig 3a, when all representative nodes run out from energy, as illustrated in Fig 3b. Unlike the EDNS algorithm, it maintains the distortion longer, because it uses the unrepresentative nodes when the representative nodes run out from energy. In such a case, we observe a better distortion level, because of the increasing in quantity of sensor nodes, as in Fig 3c, which reduces the distortion level. By assuming that each sensor harvests energy, we also observe the advantages of EDNS algorithm. But, the harvesting results some oscillations as in Fig 3d, due to that some inactive sensors are turns to be active by recovering its energy by harvesting, or vice versa, as in Fig $3 \mathrm{e}$. Beside, the network works longer than preceding case. The energy harvesting improves the network performance, because of the increment in the number of active sensor nodes, as in Fig $3 f$.

\section{CONCLUSION}

In most applications, densely deployment is required. However, it produces spatially correlated in nearby sensors, and hence their readings are redundant, which causes wasting in energy and increasing overheading packets. In this paper, we present the EDNS algorithm to address these challenges by reducing the sensors by exploiting spatial correlations between sensors through VQ. By using theoretical framework of distortion for single-hop, the accuracy is observed with respect to energy harvesting, which it is enhanced the accuracy level longer. In other word, when sensor nodes harvest energy, their life time are improved. Unlike non-harvesting case, the network losses the accuracy level when all sensors are die.

\section{ACKNOWLEDGMENT}

This work was supported by the Turkish Scientific and Technological Research Council (TUBITAK) under Grant $115 \mathrm{E} 362$.

\section{REFERENCES}

[1] I. F. Akyildiz, W. Su, Y. Sankarasubramaniam, and E. Cayirci, "Wireless sensor networks: a survey," Computer Networks, vol. 38, pp. 393-422, 2002.

[2] S. Akbari, "Energy harvesting for wireless sensor networks review," in Computer Science and Information Systems (FedCSIS), 2014 Federated Conference on, Sept 2014, pp. 987-992.

[3] M. C. Vuran and I. F. Akyildiz, "Spatial correlation-based collaborative medium access control in wireless sensor networks," IEEE/ACM Transactions on Networking, vol. 14, no. 2, pp. 316-329, April 2006.

[4] M. Gastpar and M. Vetterli, Source-Channel Communication in Sensor Networks. Berlin, Heidelberg: Springer Berlin Heidelberg, 2003, pp. 162-177. [Online]. Available: http://dx.doi.org/10.1007/3-540-36978-3 11

[5] Y. Linde, A. Buzo, and R. Gray, "An algorithm for vector quantizer design," IEEE Transactions on Communications, vol. 28, no. 1, pp. 8495, Jan 1980.

[6] T. Cover and P. Hart, "Nearest neighbor pattern classification," IEEE Transactions on Information Theory, vol. 13, no. 1, pp. 21-27, January 1967.

[7] M. Arrawatia, M. S. Baghini, and G. Kumar, "Rf energy harvesting system from cell towers in $900 \mathrm{mhz}$ band," in Communications (NCC), 2011 National Conference on, Jan 2011, pp. 1-5.

[8] W. R. Heinzelman, A. Chandrakasan, and H. Balakrishnan, "Energyefficient communication protocol for wireless microsensor networks," in System Sciences, 2000. Proceedings of the 33rd Annual Hawaii International Conference on, Jan 2000, pp. 10 pp. vol.2-. 